\title{
Clinician Survey on Speech Pathology Services for People with Aphasia in Hong Kong
}

\author{
Anthony Pak-Hin Kong ${ }^{1}$, Christine Wing-Kwan Tse ${ }^{2}$ \\ ${ }^{1}$ School of Communication Sciences and Disorders, University of Central Florida, Orlando, FL, USA; ${ }^{2}$ Department of Special Education and \\ Counselling, The Education University of Hong Kong, Hong Kong
}

This study aims to investigate aphasia practices by speech-language pathologists working with clients suffering from acquired neurogenic communication disorders through an online survey. Questions pertaining to content and intensity of aphasia management, service adequacy, and barriers to effective service provision to persons with aphasia were asked to understand the current service-provision situation in Hong Kong. A web-based questionnaire containing 38 questions was electronically distributed to speech-language pathologists in Hong Kong. A total of 41 respondents (equivalent to about $5 \%$ of the body of clinicians) completed and returned the survey. Compared to recommendations in existing best practice guidelines of aphasia from western countries, the results indicated that intensity and frequency of current aphasia services in Hong Kong fell short of expectations. Major challenges perceived by speech-language pathologists that contributed to dissatisfaction of aphasia service included limited manpower, resources, funding, and accessibility. It was also found that barriers to effective services could be related to clinicians' restricted adoption of intervention approaches, lack of active involvement of related healthcare professionals, and insufficient effort of referring persons with aphasia to support or self-help groups. Finally, factors to consider in order to improve existing aphasia management were discussed.

Keywords: Clinician survey, Aphasia, Service adequacy, Intervention approach, Best practice recommendations

\section{INTRODUCTION}

Stroke, being the fourth leading cause of death, with 3,259 (or 6.97\% of total) registered deaths [1], is a significant disease burden in Hong Kong. Based on a population health survey conducted by the Hong Kong Department of Health [2], the prevalence rates of stroke in the elderly population over 65 years was $5.5 \%$; this was greatly higher than the $0.4 \%$ prevalence in those aged between 15 and 64 years. As a rapidly ageing population, it is estimated that by the year 2036, Hong Kong will have 2.3 million people aged over 65 years [3], thus suggesting that the number of people affected by stroke will increase. One of the most common consequence of stroke is aphasia; each year, Hong Kong has over 20,000 new cases of stroke [1,4], of which up to $38 \%$ are affected by aphasia [5].

Aphasia affects a person not only in terms of communication, but also social functioning and quality of life, and is considered to be a key predictor of one's post-stroke social outcome. Compared to stroke survivors who are not affected by aphasia, persons with aphasia have been reported to participate less in activities and have a poorer qual-

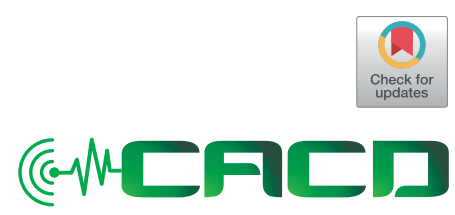

Received: September 6, 2018 Revision: December 13, 2018 Accepted: December 20, 2018

\section{Correspondence:}

Anthony Pak-Hin Kong

School of Communication Sciences and Disorders, University of Central Florida, HPA-2 106, PO Box 162215, Orlando,

FL 32816-2215, USA

Tel: +407-823-4791

Fax: +407-823-4816

E-mail: antkong@ucf.edu

(C) 2018 The Korean Association of SpeechLanguage Pathologists

This is an Open Access article distributed under the terms of the Creative Commons Attribution NonCommercial License (http://creativecommons.org/ licenses/by-nc/4.0/) which permits unrestricted noncommercial use, distribution, and reproduction in any medium, provided the original work is properly cited. 
ity of living [6]. Albeit the obvious need of post-stroke services and intervention for persons with aphasia, the focus of existing aphasia management is often placed on fast transitions between care settings or discharge planning because Speechlanguage pathologists have restricted time for providing aphasia-specific services for stroke survivors [7]. Given that Speechlanguage pathologists in acute settings tend to focus on dysphagia treatment, persons with aphasia are placed at risk of exploiting neuroplasticity during early onset and thus affect outcome of overall aphasia treatment. Moreover, there is a large array of therapy approaches addressing different aspects of deficits associated with aphasia (such as the functional approach, social/life approach, or neuropsychological approach) that can be mediated in the form of individual or group settings [8]. Choosing the best suited approach and format of intervention for persons with aphasia through individualized planning can facilitate and enhance treatment effects [9].

Speech and language services is one of the important components of stroke rehabilitation. The Hong Kong Hospital Authority has been responsible for managing public hospitals in Hong Kong and delivering services to the community since 1991. There are currently 41 public hospitals and institutions as well as 47 specialist outpatient clinics in Hong Kong managed by Hong Kong Hospital Authority. Post-stroke language therapy for persons with aphasia in the acute phases is mainly provided in government hospitals. Chronic persons with aphasia are often referred to specialist outpatient clinics for follow-up, but most of them self-refer to local university speech-language clinics, community non-profit organizations, and private clinics. In 2008, there were roughly 400 Speech-language pathologists working in Hong Kong [10] but the total number was estimated to have doubled in the past decade. According to the recent report by Hong Kong Association of Speech Therapists [11], approximately $4 \%$ of the members were employed under Hong Kong Hospital Authority and $27 \%$ registered as working in a private setting.

Speech and language intervention services for persons with aphasia in Hong Kong may be affected by cultural values and beliefs. There is a mismatch between the resources and cultural belief of receiving service among persons with aphasia in eastern and western countries [12]. For example, Asian Americans have been found to be more reticent than Americans about mental distress, thus Asian Americans will be less likely to engage in help-seeking and utilization behaviors. People with Chinese cultural value orientation exert an indirect effect on help-seeking intentions, and respond with more feelings of shame when posed with problems and thus show lower intentions to seek for help [13]. Moreover, it was reported that self-concealment was negatively related to attitude towards counseling and seeking help in the Asian and Asian American populations [14]. In other words, the difference in behavior acculturation between Asian and Western cultures contributes differently to one's willingness to look for assistance regarding personal concerns. A higher degree of public awareness of aphasia services in societies of Asian countries and better education to caregivers of persons with aphasia should, therefore, be emphasized in service delivery to ensure that all persons with aphasia can receive treatment without feeling the need for self-concealment.

In Hong Kong, research on speech and language pathology is limited and its related information is generally less accessible compared to western countries, which are known for their comprehensive and funding-supported provision of education, training, and services for individuals with communication disorders [15]. Kong [16] is one of the very few reports on a family-member survey regarding rehabilitation services for persons with aphasia in Hong Kong. The results revealed that aphasia treatments were insufficient in terms of duration (over $50 \%$ of sessions lasted for only 30 minutes or shorter) and frequency (acute persons with aphasia only attended weekly and chronic persons with aphasia attended monthly session), which was below the standards suggested by most existing best practice guidelines (e.g., [17]). A high proportion of respondents $(>42 \%$ ) were dissatisfied with the aphasia services they had received although the content and approaches of treatment utilized had not been specified.

\section{Aims}

To optimize client care and service delivery for persons with aphasia, knowledge of current practices regarding assessment as well as post-stroke rehabilitation and intervention is required. At present, little information is available regarding the current practice of aphasia therapy in Hong Kong. This study aims to investigate aphasia practices by Speech-language pathologists working with clients suffering from acquired neurogenic communication disorders through an online survey. Specifically, questions pertaining to the content and intensity of aphasia management, adequacy of services, and barriers to effective service provision to persons with aphasia (in the context of best practice guidelines in most western countries) were asked in the clinician survey to understand the current service-provision situation in Hong Kong. 
It is anticipated that the results of this study will potentially allow for reallocation of resources by the service providers to facilitate aphasia rehabilitation, and to allow local clinicians to gain a clearer picture of current service provision compared to the ideal management of aphasia, thus to identify how assessment and intervention practices can be modified, and in turn to improve the quality of living for persons with aphasia.

\section{METHODS}

\section{Survey design}

The survey was designed to include major parameters guidelines of service delivery for persons with aphasia to allow for comparison between actual and recommended practice. A pilot survey was completed by five student Speech-language pathologists at The Education University of Hong Kong. With reference to the received feedback regarding the clarity, length, and appropriateness of the questions, the survey was finalized and consisted of 38 items covering the following topics, including (i) Background information, i.e., work setting and clinical experience in aphasia of the SLP respondents, (ii) Aphasia assessment, e.g., assessment tools, outcome measures, and frequency and duration of assessment sessions for persons with aphasia, (iii) Service delivery/Treatment, e.g., frequency of sessions for persons with aphasia, average number of sessions per persons with aphasia, average duration of treatment session, and intervention content utilized, (iv) Aphasia intervention approaches, e.g., clinician's knowledge of different aphasia treatment approaches and their level of confidence and frequency of using these approaches, (v) Client education and counselling, e.g., frequency of client education and counselling, methods of providing client education, and frequency of community education activities, and (vi) Goal setting and discharge, e.g., frequency of collaborating with persons with aphasia and family in goal-setting, reason(s) for discharge, and content of review sessions. Survey Gizmo was used to develop the online questionnaire. Details of the 38 questions, which included answer formats of Yes/No, rating scales, checklists and open-ended text boxes, are listed in Appendix A.

To assess the reliability of self-rating scales pertaining to knowledge, confidence and frequency of using approaches to aphasia rehabilitation, reliability analyses were conducted. The knowledge scale consisted of 11 items $(\alpha=0.88)$, the confidence scale consisted of 11 items $(\alpha=0.86)$, and the frequency scale consisted of 11 items $(\alpha=0.6)$. Knowledge and confidence scales had good internal consistency whilst frequency scales had acceptable internal consistency. The measures also had good face validity.

\section{Procedures}

A web-questionnaire was distributed to practicing Speechlanguage pathologists in Hong Kong working in hospitals, private settings, university clinics, non-profit organizations, and nursing homes. The questionnaire was electronically released and participants were recruited via email, social media platforms, and through listing on the Monthly Newsletter of the Hong Kong Association of Speech Therapists.

\section{Statistical analysis}

The data from Survey Gizmo was downloaded and collated into an Excel database to ensure valid responses and to rule out duplicate responses. Descriptive statistics and correlational analyses was generated. Anchor points was given to questions utilizing 5-point rating scales. Responses to openended questions was downloaded to perform content analysis. Results from the descriptive review of present study were then used to analyze survey results.

\section{RESULTS}

\section{Participants}

A total of 41 Speech-language pathologists in Hong Kong, with an accumulated clinical experience of providing services to persons with aphasia of 7.21 years (s.d. $=5.24$ years), completed the survey. The distribution of their primary work setting was as follow: Hospitals (39\%), Private clinics (24\%), University clinics (20\%), and non-profit organizations (17\%), thus covering a broad spectrum of working environments. Over half of the respondents had $10 \%$ to $50 \%$ of caseload involving persons with aphasia. However, the dominant caseload of Speech-language pathologists in the hospital setting was reported to be clients with dysphagia.

\section{Service delivery}

Concerning assessment of aphasia, most of our respondents reported an average duration of 60 minutes $(41.5 \%)$ or 30 to 45 minutes (36.6\%) per session. Interesting, out of the four clinical settings, only Speech-language pathologists in the hospital group reported providing assessment in 30-minute sessions (21.9\%). Information regarding assessment batteries utilized was also sought. A total of 11 assessment/screening 
tools were reported, such as the Cantonese version of Western Aphasia Battery [18] (50.0\%), Cantonese Linguistic Communication Measure [19] (11.8\%), Psycholinguistic Assessments of Language Processing (3.9\%), Clinical Evaluation of Language Fundamentals (2.6\%), or The Multimodal Communication Screening Test for Aphasia (2.6\%). Use of informal assessment tools (18.4\%) or self-developed items (6.6\%) was not uncommon. In addition, $14.6 \%$ of the SLP respondents reported to have made revisions to the existing English aphasia screening/assessment tools for their Cantonese-speaking clientele in Hong Kong. Nearly all the tools listed by our Speech-language pathologists were impairment-based assessment batteries and only $1 \%$ reported was specific for cognitive-communication disorders (i.e., the Montreal Cognitive Assessment). Interestingly, there were no mentioning of measures that tapped on persons with aphasia's functional communication, language sampling, or quality of life.

As for questions related to aphasia intervention, it was found that the majority of Speech-language pathologists provided treatment once per month (48.8\%). Again, hospitalbased clinicians tended to provide the shortest treatment sessions of less than 30 minutes at $68.8 \%$ of the time. With regards to types of treatment provided, all Speech-language pathologists reported to have included individual therapy in sessions. Communication partner training was the second most included intervention, followed by group aphasia therapy and social community education. None of our respondents reported provision of other types of intervention, such as telerehabilitation, computer- or technology-based therapy, in their treatment sessions.

\section{Rehabilitation approaches}

The respondents self-rated their knowledge on, confidence with, and frequency of using eleven different approaches in aphasia rehabilitation, including (i) Cognitive Neuropsychological approach, (ii) Functional approach, (iii) Social/life participation, (iv) Discourse-based intervention, (v) Technology-based intervention, (vi) Stimulation, (vii) Group therapy, (viii) Principles of neuroplasticity, (ix) Augmentative and alternative communication, ( $\mathrm{x}$ ) Conversation partner training, and (xi) Training for culturally and linguistically diverse persons with aphasia (see Appendix A for details). Results are displayed in Figure 1.

Speech-language pathologists' knowledge on and confidence with 'Functional, 'Social/life participation', and 'Principles of neuroplasticity' were rated the highest. These three ap- proaches were also reported to be the most frequently used. Knowledge and confidence of 'Technology-based, 'Stimulation' and 'Training for culturally and linguistically diverse populations' were rated as 'Limited' or 'Unconfident' by most respondents; they were reported to be used only 'Sometimes' by over half of the respondent, who self-claimed to be 'very knowledgeable' of and 'very confident' on them.

Pearson Correlation test of independence was calculated to determine if the Speech-language pathologists' knowledge of an approach was independent to their confidence level in clinical application. Moderate and positive interactions were found in all approaches with high significant levels. The Pearson Correlation test of independence was also calculated to determine whether clinicians' knowledge of and confidence of different interventions was related to the frequency in implementation. In terms of knowledge and frequency of use, as well as confidence and frequency of use, only weak to moderate interactions were found for cognitive neuropsychological, social/life participation, principles of neuroplasticity, augmentative and alternative communication, conversation partner training, and training for culturally and linguistically diverse populations (Table 1).

\section{Client education}

The respondents were asked about the frequency of and methods used in educating persons with aphasia, their caregivers, and other health practitioners about aphasia as an integral part of clinical responsibility. A relatively small percentage of Speech-language pathologists 'always' provided education, to persons with aphasia (22\%) and caregivers (29.2\%). In contrast, $63 \%$ of respondents reported doing so 'frequently' or 'occasionally' to health practitioners (only $2.4 \%$ of them indicated they 'never' provided client education to other health practitioners). Concerning the methods adopted for education, all participants (100\%) reported the use of verbal means in giving relevant information or outlining options of aphasia treatment to both caregivers and persons with aphasia. The next two popular methods were through written $(63.4 \%)$ and web-based materials (48.8\%). Only $4.9 \%(n=4)$ reported their use of providing demonstrations in the education process.

\section{Goal setting and discharge}

The results revealed extensive involvement of persons with aphasia and caregivers when Speech-language pathologists set intervention goals. Specifically, Speech-language pathologists 'always' (24.9\%) and 'frequently' (53.7\%) collaborated 

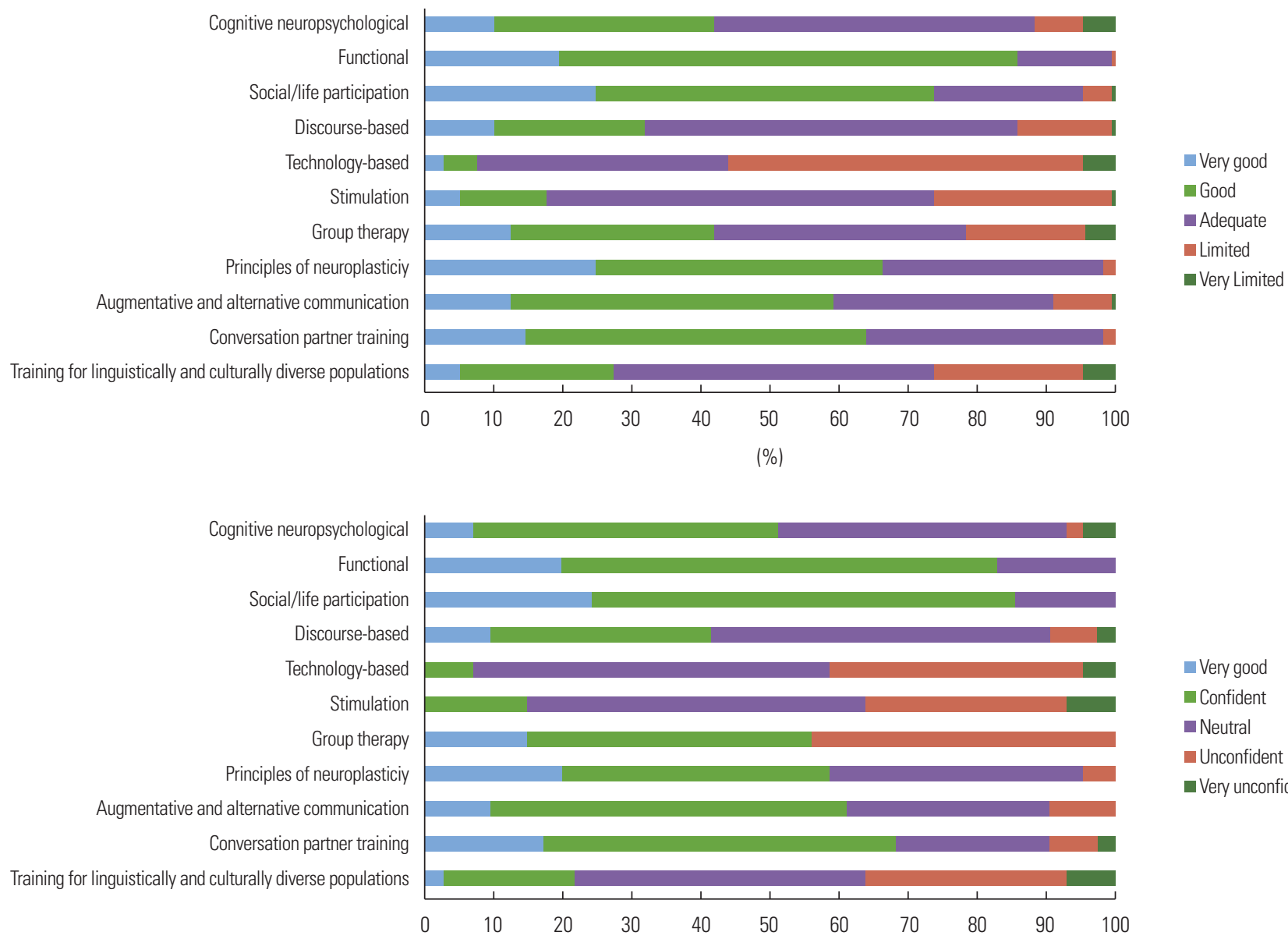

$(\%)$

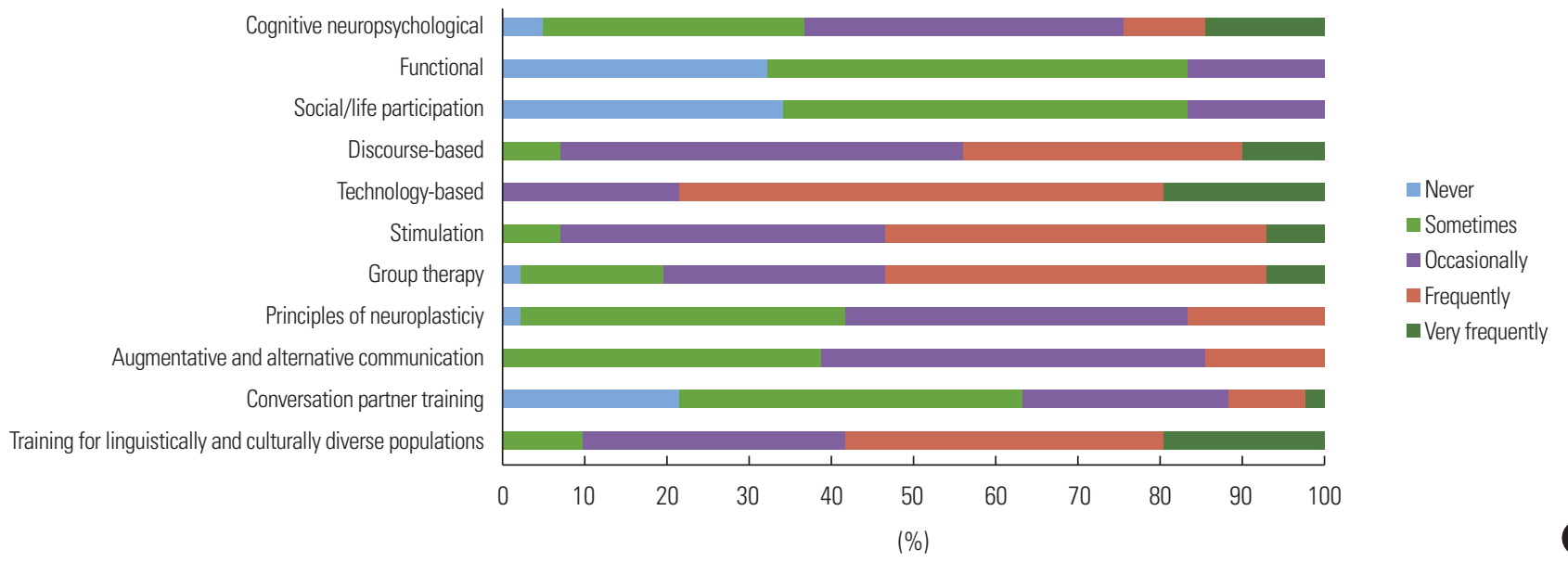

Figure 1. Percentage of participants' ratings on (A) knowledge, (B) confidence and (C) frequency of use of different approaches to aphasia therapy.

with persons with aphasia when setting goals. This was also the case for involving caregivers: 'always' $(22.0 \%)$ and on a 'frequent' basis (68.3\%),

In regard to discharging persons with aphasia from therapy, the most frequently reported reason was 'poor' or 'plateaued' progress in therapy sessions (18.4\%), followed by clients achieving functional communication (14.5\%), and poor prognosis of the persons with aphasia (13.2\%). Other reasons in- 
Table 1. Correlational relationships between knowledge on, confidence with, and frequency of using various approaches to aphasia therapy

\begin{tabular}{lccc}
\hline \multirow{2}{*}{ Intervention type/Approach } & \multicolumn{3}{c}{ Pearson's $R$} \\
\cline { 2 - 3 } & \multicolumn{1}{c}{ Knowledge and confidence of use } & Knowledge and frequency of use & Confidence and frequency of use \\
\hline Cognitive neuropsychological & $0.629^{* *}$ & $0.456^{* *}$ & $0.401^{*}$ \\
Functional & $0.69^{* *}$ & 0.287 & 0.227 \\
Social/life participation & $0.65^{* *}$ & $0.437^{* *}$ & $0.456^{* *}$ \\
Discourse-based & $0.544^{* *}$ & 0.165 & 0.081 \\
Technology-based & $0.539^{* *}$ & 0.185 & 0.075 \\
Stimulation & $0.414^{* *}$ & 0.064 & 0.04 \\
Group therapy & $0.655^{* *}$ & 0.159 & 0.159 \\
Principles of neuroplasticity & $0.649^{* *}$ & $0.499^{* * *}$ & $0.45^{* *}$ \\
Augmentative and alternative & $0.477^{* *}$ & $0.397^{*}$ & $0.597^{* * *}$ \\
communication & & & $0.307^{*}$ \\
Conversation partner training & $0.639^{* *}$ & $0.587^{* * *}$ & $0.329^{*}$ \\
Training for linguistically and culturally & $0.643^{* *}$ & $0.402^{*}$ & \\
diverse populations & & &
\end{tabular}

cluded client withdrawal or health reasons, low motivation of persons with aphasia, limited financial support, and deficits were minor. Interestingly, $100 \%$ of the respondents indicated that they believed their persons with aphasia clients still required language intervention at the time of discharge and, therefore, home-practice was always given (100\%). Other common recommendations given by Speech-language pathologists included 'educational materials' (63.4\%) and referral to community self-help groups (41.5\%) or hospital-/nonprofit organizations-based support groups (48.8\%).

In addition, $14.6 \%, 36.6 \%$, and $34.1 \%$ of Speech-language pathologists reported they 'frequently,' 'occasionally', or 'never,' respectively, reviewed their patients after discharge. Only $1 \%$ of them reported their persons with aphasia clients were 'always' reviewed. Of the respondents who provided follow-up to their persons with aphasia $(n=27)$, the most frequently reported review-interval was at ' 6 months' ( $40.7 \%)$, followed by ' 1 year' (22.2\%) and 'over 1 year' (18.5\%). Only a small portion of persons with aphasia were reviewed shortly after discharge: $14.8 \%$ in ' 2 to 3 month' and $3.7 \%$ in ' 2 weeks to 1 month' intervals. Concerning the content of the follow-up sessions, it was found that while Speech-language pathologists always reviewed the persons with aphasia's language ability (100\%), language training for persons with aphasia and communication partner training was also conducted by $66.7 \%$ of Speechlanguage pathologists.

\section{Challenges and facilitators to service delivery for persons with aphasia}

Our participants were asked to rate their overall satisfaction with the current service delivery for persons with aphasia in Hong Kong; 12\% indicated 'very unsatisfied', $44 \%$ were unsatisfied, and the remaining $44 \%$ were neutral. With reference to the open-ended question related to Speech-language pathologists' view on perceived barriers and facilitators to service delivery for persons with aphasia in Hong Kong, the top four listed barriers to effective aphasia services were limited manpower (17.7\%), limited resources (15.6\%), limited government funding (13.3\%), and limited accessibility (13.3\%). Other reported barriers included the lack of evidence-based practice for Hong Kong populations (11.1\%), limited time for sessions (11.1\%), low client motivation (6.7\%), limited involvement for persons with aphasia influenced by the culture in Hong Kong (4.4\%), and limited support from caregivers (4.4\%).

Our Speech-language pathologists specified that increasing the amount of clinical resources or tools tailored for the Hong Kong population (41.7\%) would be the top facilitator to enhancing aphasia services. Other suggestions included additional funding to support clinical services (18.8\%), employing more Speech-language pathologists (16.7\%), enhancing clients' compliance of home practice $(8.3 \%)$, more frequent treatment to persons with aphasia (4.2\%), increasing patient motivation (4.2\%) and caregiver support (4.2\%), and providing resources for persons with aphasia's vocational training $(2.1 \%)$. 


\section{DISCUSSION}

This study is the first report on current speech pathology practice specific to persons with aphasia in Hong Kong and revealed findings with considerable implications to practice planning and future research in aphasia rehabilitation. It is estimated that our respondents represented $5 \%$ of the local Speech-language pathologists body practicing in Hong Kong. In particular, the possible discrepancy in adequacy of service delivery for persons with aphasia between Hong Kong and countries in the West, as we discuss below in detail, would ensure local Speech-language pathologists and Government to better understand the present status of service provision. We hope that this would potentially lead to modification of management programs through comparison to countries that excel.

Unsurprisingly, Speech-language pathologists in Hong Kong faced the same challenge as clinicians in the west, in terms of insufficient clinical resources and materials to support their practice. This can be considered as a major barrier to effective aphasia service delivery. Note that the assessment tools our respondents commonly used were mainly the ones that are impairment-based. This may potentially suggest that other impacting factors on the persons with aphasia's functional communication, such as life activity or social participation, were less holistically considered. In the past few years, a number of newly developed assessment batteries that are linguistically sensitive and culturally suitable have been published; some examples included the Hong Kong version of the Oxford Cognitive Screen [20], Cantonese version of Birmingham Cognitive Screen [21,22], Main Concept Analysis [23,24]. These tools can hopefully broaden the practice scope and widen the resource base of clinical diagnosis of aphasia in Hong Kong.

Given that evidence-based treatment protocols geared for native persons with aphasia in Hong Kong are still lacking, more research is warranted to allow us to better address barriers and gaps of service provision and to enhance the social living and quality of life of persons with aphasia. It has been recommended that aphasia treatment should best be provided five days per week or as intensive as tolerated [25,26]. Our results showed a clear insufficiency in terms of the frequency of aphasia training currently being offered (e.g., once weekly in university and private clinics, which was the closest to recommended level). This may suggest that paid services have potential influence on how often treatment can be provided to clients. Moreover, the result regarding duration of hospital-based aphasia rehabilitation (sessions of 30 minutes) was also found to be below the recommended standard of 45 to 60 minutes [27]. More manpower, resources, and funding, therefore, need to be given to acute/outpatient hospitals to allow service delivery that will be comparable to the international standard. Like treatment for any other speech-language disorder, treatment for aphasia is also individualized. However, it appears that our respondents tended to be restrictive in this aspect and the majority of treatment sessions were conducted through individual training. Finally, more efforts can be paid on targeting the improvement of communication access and reduction of stigma among persons with aphasia, especially because the difference of western and eastern cultures will affect service delivery for persons with aphasia. Kucukarslan [28] has explained why clients would decline treatment due to their perception of language and communication problems as these deficits related to cultural influences on prescribed treatments.

Concerning the caseload of clinicians surveyed, it was found that the dominant caseload in the hospital settings was dysphagia. This is in contrast to the report by Rose et al. [29] in which aphasia dominated the inpatient, outpatient, and community level rehabilitation settings. What also needs to be highlighted is that Speech-language pathologists working in settings of non-profit organizations or private clinics reported a high caseload of pediatric over adult/geriatric cases. This may be reflective of the lower degree of awareness regarding aphasia in the Chinese population and the different cultural values of Chinese who are more reluctant to seek help when posed with problems [13].

The high ratings of knowledge about and confidence in adopting functional and social-life participation approaches of aphasia rehabilitation as well as principles of neuroplasticity reported by clinicians in Hong Kong were similar to reports in Australia [29] and Singapore [30]. This suggests Speech-language pathologists' considerations of life participation under the WHO-ICF model, despite the lack of comprehensiveness in assessment procedures. The positive correlation between the clinicians' knowledge and confidence in using each treatment approach also reflects that continued research and development of related approaches in Hong Kong may facilitate local Speech-language pathologists' ability to extent existing therapy. In addition, our results revealed that the therapeutic approaches rated the highest in terms of knowledge and confidence by the respondents were most frequently used at the same time (despite the lack of significant 
correlation between knowledge/confidence and frequency of application of approaches to aphasia therapy in general). This seems to suggest that clinicians decide on which approach to utilize based on client's ability rather than their knowledge/ confidence in application.

The present finding of Speech-language pathologists' selfrating of knowledge on and level of comfort in applying various clinical approaches of aphasia rehabilitation also sheds some light on direction of continuing education for Speechlanguage pathologists. It was clear that as compared to impairment-based intervention, local clinicians were less equipped with knowledge base for managing the culturally and linguistically diverse populations. Knowing that $11.9 \%$ of the population in Hong Kong do not speak Cantonese as their mother tongue [3], with the percentage excepted to continually increase, local Speech-language pathologists may face more challenges for future serving of this potentially growing caseload. Equally significant will be the expansion of Speechlanguage pathologists' foundations of knowledge, such as persons with aphasia's social communication [31], discourse training, or group interventions that target social interactions, and their staying up-to-date on new developments, such as tele-rehabilitation to improving accessibility [32,33]. In light of the high penetration and easy access of tablets, smart devices, and internet in Hong Kong [3], technology-based aphasia therapy, e-learning, or i-Rehabilitation will become an important facilitator to service delivery.

One of the keys to successful aphasia management centers around educating persons with aphasia, their families, and related healthcare about aphasia through receive verbal and/ or written materials $[17,26,34-36]$. The results from the survey suggests that there is room for improvement on this aspect where local Speech-language pathologists can strategically collaborate with different professionals when they provide services to persons with aphasia. Moreover, Speech-language pathologists may also consider utilizing a more diverse range of educational materials to persons with aphasia and their caregivers, instead of solely adopting a client-based approach in the process; this can be on top of the component of counselling $[37,38]$.

According to the British Department of Health [39], it is recommended that persons with aphasia be reviewed 6-weeks from discharge. However, our results indicated that this was not the usual practice in Hong Kong (e.g., the majority of persons with aphasia follow-up sessions were provided 6 months to 1 year after discharge). This is worrying because Speech- language pathologists are limited in monitoring maintenance and/or regression of different acquired treatment effects among persons with aphasia, and subsequently cannot provide them with appropriate remediation. The beneficial effects of pairing persons with aphasia with support groups upon discharge have been documented $[26,40]$. Yet, our current services for persons with aphasia during this transition period seemed to be inadequate because only about $50 \%$ of Speech-language pathologists referred their persons with aphasia to self-help or support groups once the therapy is terminated. Moreover, not all persons with aphasia received updated information regarding their functional abilities and/or materials as well as related resources for continuing rehabilitation upon discharge.

\section{Limitations and future directions}

There are several limitations of the present study. First, our comparisons of 'best practice' for aphasia versus that of Speech-language pathologists in Hong Kong was based on a small number of countries due to lack of relevant research done on this topic in countries such as Australia [41], USA [42] or United Kingdom [43,44]. The findings may also not be readily generalizable to service delivery in underdeveloped countries. Due to differences in culture beliefs, government funding systems, cultural diversity, and how aphasia intervention is prioritized in Asian countries, one may question how easily applicable our survey results are to other Asian populations. Future directions in extending the current study may include examination of practice for other communication disorders beyond aphasia; this can lead to a clearer idea of how the field of speech and language pathology is developing in Hong Kong. Additionally, clinicians in Hong Kong are most concerned about locally-relevant resources in serving persons with aphasia, which warrants continuing research in developing these said resources for more holistic rehabilitation. With the upcoming trend of applying tele-rehabilitation and advancement of technology in aphasia management in Hong Kong, one may investigate how clinical service for persons with aphasia will improve using technology.

\section{CONCLUSIONS}

Speech and language services is one of the important components of post-stroke rehabilitation for speakers with aphasia. At present, little information regarding the current practice of aphasia therapy in Hong Kong is available. Being the first cli- 
nician survey on current speech pathology services offered specifically to aphasia in Hong Kong. Over half of our respondents (representing $5 \%$ of local body of practicing speech and language pathologists) were dissatisfied with the service delivery for speakers with aphasia. Existing practice fell short of most western standards in terms of manpower and financial resources as well as knowledge base of managing aphasia in Chinese. Replication of this study to other Asian countries will allow us to examine the quality of speech and language services in various cultural contexts.

\section{Declaration of interest statement}

The authors report no conflicts of interest. Both authors contributed to the research design, acquisition and interpretation of data, as well as drafting, amending, and critically reviewing this manuscript.

\section{REFERENCES}

1. Hong Kong Department of Health. Health facts of Hong Kong. Hong Kong: Department of Health; 2016.

2. Hong Kong Department of Health. Report on population health survey 2003/2004. Hong Kong: Department of Health; 2005.

3. Hong Kong Department of Census and Statistics. Thematic Household Survey Report. 2016;59:44-88.

4. Hong Kong Hospital Authority. Hospital Authority annual report 2014-2015. Hong Kong: Hong Kong Hospital Authority; 2016.

5. Salter K, Teasell R, Foley N, Allen L. The evidence-based review of stroke rehabilitation (EBRSR) reviews current practices in stroke rehabilitation. Available from EBRSR.com.

6. Hilari K. The impact of stroke: are people with aphasia different to those without? Disability and Rehabilitation. 2011;33:211-218.

7. Hersh D. Therapy in transit: managing aphasia in the early period post stroke. Aphasiology. 2016;30:509-516.

8. Martin N, Thompson CK, Worrall L. Aphasia rehabilitation: The impairment and its consequences. Plural Publishing; 2007.

9. Damasio AR. Aphasia. New England Journal of Medicine. 1992; 26:531-539.

10. Hong Kong Association of Speech Therapists. Comments on the Healthcare Reform submitted by the Hong Kong Association of Speech Therapists. Hong Kong: Available from http://www.fhb. gov.hk/beStrong/files/organizations/O082.pdf; 2017.

11. Hong Kong Association of Speech Therapists. Annual report 20152016. Hong Kong. Available from https://speechtherapy.org.hk/ Documents.html; 2017.

12. Zhang AY, Snowden LR, Sue S. Differences between Asian and White Americans' help seeking and utilization patterns in the Los Angeles area. Journal of Community Psychology. 1998;26:317-326.

13. Lau A, Takeuchi D. Cultural factors in help-seeking for child behavior problems: value orientation, affective responding, and se- verity appraisals among Chinese-American parents. Journal of Community Psychology. 2001;29:675-692.

14. Liao HY, Rounds J, Klein AG. A Test of Cramer's (1999) Help-seeking model and acculturation effects with Asian and Asian American college students. Journal of Counseling Psychology. 2005;52: 400-411.

15. Cheng LR. Emerging issues in health and education in Asia-Pacific: a focus on speech-language pathology. Folia Phoniatrica et Logopaedica. 2010;62:238-245.

16. Kong APH. Family members' report on speech-language pathology and community services for persons with aphasia in Hong Kong. Disability and Rehabilitation. 2011;33:2633-2645.

17. Aphasia United. Aphasia United best practice recommendations for aphasia. Available from http://www.aphasiaunited.org/bestpractice-recommendations/.

18. Yiu EML. Linguistic assessment of Chinese-speaking aphasics: development of a Cantonese aphasia battery. Journal of Neurolinguistics. 1992;7:379-424.

19. Kong APH, Law SP. A linguistic communication measure for monitoring changes in Chinese aphasic narrative production. Clinical Linguistics and Phonetics. 2009;23:255-269.

20. Kong APH, Lam PHP, Ho DWL, Lau JK, Humphreys G, Riddoch J, et al. The Hong Kong version of the Oxford Cognitive Screen (HKOCS): Validation study for Cantonese-speaking chronic stroke survivors. Aging, Neuropsychology, and Cognition. 2016;23: 530548.

21. Kong APH, Chan J, Lau JKL, Bickerton WL, Weekes B, Humphreys G. Developing a Cantonese version of Birmingham Cognitive Screen for stroke survivors in Hong Kong. Communication Disorders Quarterly. 2017;39:387-401.

22. Pan X, Chen H, Bickerton WL, Lau JKL, Kong APH, Rotshtein P, et al. Preliminary findings on the reliability and validity of the Cantonese Birmingham Cognitive Screen in patients with acute ischemic stroke. Neuropsychiatric Disease and Treatment. 2015;11: 2377-2390.

23. Kong APH. The main concept analysis in Cantonese aphasic oral discourse: external validation and monitoring chronic aphasia. Journal of Speech, Language, and Hearing Research. 2011;54:148159.

24. Kong APH. The Main Concept Analysis (MCA) for oral discourse production. Hong Kong: The Commercial Press (H.K.) Limited; 2016.

25. Lindsay P, Bayley M, Hellings C, Hill M, Woodbury E, Phillips S. Canadian best practice recommendations for stroke care (updated 2008). Canadian Medical Association Journal. 2008;179:S1-S25.

26. Australian Aphasia Rehabilitation Pathway. Aphasia rehabilitation best practice statement. Australia: Queensland; 2014.

27. Godecke E, Ciccone NA, Granger AS, Rai T, West D, Cream A, et al. A comparison of aphasia therapy outcomes before and after a Very Early Rehabilitation programme following stroke. International Journal of Language \& Communication Disorders. 2014;49: 149-161.

28. Kucukarslan SN. A review of published studies of patients' illness 
perceptions and medication adherence: lessons learned and future directions. Research in Social and Administrative Pharmacy. 2012;8:371-382.

29. Rose M, Ferguson A, Power E, Togher L, Worrall L. Aphasia rehabilitation in Australia: current practices, challenges and future directions. International Journal of Speech-Language Pathology. 2014;16:169-180.

30. Guo YE, Togher L, Power E. Speech pathology services for people with aphasia: what is the current practice in Singapore? Disability and Rehabilitation. 2014;36:691-704.

31. Worrall L, Serrate S, Rogers P, Howe T, Hersh D, Ferguson A, et al. What people with aphasia want: their goals according to the ICF. Aphasiology. 2011;25:309-322.

32. Hill AJ, Theodoros DG, Russell TG, Ward EC, Wootton R. The effects of aphasia severity on the ability to assess language disorders via telerehabilitation. Aphasiology. 2009;23:627-642.

33. Georgeadis A, Brennan D, Barker L, Baron C. Telerehabilitation and its effect on story retelling by adults with neurogenic communication disorders. Aphasiology. 2004;18(5-7):639-52.

34. Canadian Stroke Network. Canadian best practice recommendations for stroke care. Canada: Ottawa; 2010.

35. Hebert D, Lindsay MP, McIntyre A, Kirton A, Rumney PG, Bagg S, et al. Canadian stroke best practice recommendations: stroke rehabilitation practice guidelines, update 2015. International Journal of Stroke. 2016;11:459-484.

36. Simmons-Mackie N, Raymer A, Armstrong E, Holland A, Cherney LR. Communication partner training in aphasia: a systematic re- view. Archives of Physical Medicine and Rehabilitation. 2010;91: 1814-1837.

37. Cunningham R. Counselling someone with severe aphasia: and explorative case study. Disability and Rehabilitation. 1998;20:346354.

38. Simmons-Mackie N, Damico JS. Counseling and aphasia treatment: missed opportunities. Topics in Language Disorders. 2011; 31:336-351.

39. Department of Health. National stroke strategy. London: DH. Available from http://webarchive.nationalarchives.gov.uk/+/ http://www.dh.gov.uk/en/Healthcare/Longtermconditions/Vascular/Stroke/DH_099065

40. Lanyon LE, Rose ML, Worrall L. The efficacy of outpatient and community-based aphasia group interventions: a systematic review. International Journal of Speech Language Pathology. 2013; 15:359-374.

41. Foster A, O'Halloran R, Rose M, Worrall L. Communication is taking a back seat: Speech pathologists' perceptions of aphasia management in acute hospital settings. Aphasiology. 2016;30:585-608.

42. Garcia JM, Garrett KL, Pimentel JT, Garcia RG. Clinical services for aphasia: a survey of university clinics. Aphasiology. 2002;16:715726.

43. Code C, Heron C. Services for aphasia, other acquired adult neurogenic communication and swallowing disorders in the United Kingdom, 2000. Disability and Rehabilitation. 2003;25:1231-1237.

44. Code C, Petheram B. Delivering for aphasia. International Journal of Speech Language Pathology. 2011;13:3-10. 


\section{Appendix A}

\section{Questions in the clinician survey for examining the current speech pathology practices for persons with aphasia in Hong Kong}

\section{Section A: Background Information}

1. Are you currently working in the speech-pathology field? [Yes/No]

2. What is your current primary work setting? [Hospital/Private practice/non-profit organizations/Nursing home/University clinic/Other (please state)]

3. What is the dominant caseload in your current work setting? [Dysphagia/Apraxia of Speech or Dysarthria/Aphasia/Cognitive communicative disorders/Other (please state)]

4. In the course of your practice, what is the approximate percentage of caseload that contains people with aphasia? $[<10 \%, 10-25 \%, 26-50 \%, 51$ $75 \%,>75 \%]$

5. How long is your accumulated clinical experience with aphasia (months/years)? [__years __months]

\section{Section B: Assessment/Screening}

6. Please list the screening/assessment tools you have used for persons with aphasia.

7. What is the average duration of an assessment session you provide to persons with aphasia? [<30 minutes, $30-45$ minutes, 1 hour, 1.5 hours, $>1.5$ hours]

8. Have you made revisions to screening/assessment tools for aphasia? [Yes/No-Go to Q12]

9. How often do you need to reevaluate and/or revise screening/assessment tools of aphasia? [Less frequent than once per 3 year/Once every 3 years/Yearly/Once every 6 months/More frequent than once per 6 months]

10. What screening/assessment tools of aphasia have you reevaluated and/or revised?

11. What revisions did you make (tick all that apply)? [Translation/Omission of items/Adding items/Cueing hierarchy/Changing instructions/ Other (please state)]

\section{Section C: Service delivery/Treatment}

12. What is the average frequency of treatment sessions you provide to persons with aphasia? [Daily/More than once per week/Weekly/Bi-weekly/Monthly]

13. What is the average duration of a treatment session you provide to persons with aphasia? $[<30$ minutes, $30-45$ minutes, 1 hour, 1.5 hours, $>1.5$ hours]

14. What intervention do you provide to persons with aphasia (tick all that apply)? [Individual therapy/Group therapy/Communication partner training/Telerehabilitation/Social community education/Other (please state)]

15. Please list any challenges you face in provision of effective aphasia intervention services (For example, limited resources/funding, accessibility, lack of strong scientific evidence, etc.).

16. Please list factors that can facilitate your service provision for persons with aphasia.

17. How satisfied are you with the current service delivery provided for persons with aphasia? [Very dissatisfied/Dissatisfied/Neutral/Satisfied/ Very satisfied/]

18. Which of the following areas should be improved for aphasia service delivery (tick all that apply)? [Frequency of sessions/Duration of sessions/Community education/Number of sessions in a block/Communication partner training/Other (please state)]

\section{Section D: Clinical approaches of aphasia rehabilitation}

Characteristics of different clinical approaches of aphasia rehabilitation are given below:

\begin{tabular}{|l|l|l|}
\hline i. & $\begin{array}{l}\text { Cognitive Neuropsychological ap- } \\
\text { proach }\end{array}$ & $\begin{array}{l}\text { Addressing language impairment with reference to information processing models of language production and } \\
\text { comprehension; e.g., Semantic based treatments, phonological based treatments }\end{array}$ \\
\hline ii. & Functional approach & $\begin{array}{c}\text { Focusing on improving performance on everyday communicative tasks such as ordering a meal, asking for help; } \\
\text { e.g., Script training }\end{array}$ \\
\hline iii. & Social/life participation & $\begin{array}{c}\text { Engaging persons with aphasia in goal setting and focusing on specific social relationships and facilitate persons } \\
\text { with aphasia's participation in society; e.g., strengthening daily participation in activities of choice }\end{array}$ \\
\hline iv. & Discourse-based intervention & Use of narrative, expository and conversational discourse; e.g., Main concept analysis; T-units \\
\hline v. & Technology-based intervention & Use of telerehabilitation, videos, recordings, computer programs or applications; e.g., AphasiaScripts, ORLA \\
\hline
\end{tabular}




\begin{tabular}{|l|l|l|}
\hline vi. & Stimulation & $\begin{array}{c}\text { Using intense auditory and visual stimulation and task hierarchies to stimulate language modalities and elicit } \\
\text { responses; e.g., Schuell's Stimulation Approach, MIT }\end{array}$ \\
\hline vii. & Group therapy & Conducting therapy with small groups of persons with aphasia \\
\hline viii. & Principles of neuroplasticity & $\begin{array}{c}\text { Considering principles of neuroplasticity and taking into account of treatment intensity, salience of stimuli, } \\
\text { schedule of feedback/practice; e.g., Constraint induced language treatment }\end{array}$ \\
\hline ix. & $\begin{array}{l}\text { Augmentative and alternative } \\
\text { communication }\end{array}$ & $\begin{array}{c}\text { Providing external support for persons with aphasia who cannot understand/generate messages on their own } \\
\text { to maximize functional communication; e.g., communication boards/devices, gesturing, writing }\end{array}$ \\
\hline x. & $\begin{array}{l}\text { Conversation partner training } \\
\text { Training persons with aphasia's communication partner(s) with strategies designed to improve communication } \\
\text { participation and effectiveness of persons with aphasia; e.g., conversational coaching, SPPARC training }\end{array}$ \\
\hline xi. & $\begin{array}{l}\text { Training for culturally and linguis- } \\
\text { tically diverse persons with } \\
\text { aphasia }\end{array}$ & $\begin{array}{c}\text { dddressing communication of persons with aphasia who come from different backgrounds or speak languages } \\
\text { other than Cantonese }\end{array}$ \\
\hline
\end{tabular}

19. How would you rate your knowledge in each of the above clinical approaches of aphasia rehabilitation? [Very good/Good/Adequate/Limited/Very limited]

20. How would you rate your current level of confidence in providing service to persons with aphasia using the above clinical approaches of aphasia rehabilitation? [Very confident/Confident/Neutral/Unconfident/Very unconfident]

21. How often do you use the above clinical approaches of aphasia rehabilitation in your practice? [Very frequently /Frequently /Sometimes/ Rarely /Very rarely]

\section{Section E: Client education and counselling}

22. Do you educate persons with aphasia about aphasia? [Always/Frequently/Occasionally/Rarely/Never]

23. What do you use to educate persons with aphasia (tick all that apply)? [Verbal explanation/Written information/Video resources/Web-based material/Other (please state)]

24. Do you educate the persons with aphasia's family and communication partners about aphasia? [Always/Frequently/Occasionally/Rarely/ Never]

25. What do you use to educate the persons with aphasia's family and communication partners about aphasia (tick all that apply)? [Verbal explanation/Written information/Video resources/Web-based material/Other (please state)]

26. Do you provide counselling to persons with aphasia and family regarding the emotional and social changes that may occur with aphasia? [Always/Frequently/Occasionally/Rarely/Never]

27. Do you perceive yourself as competent in providing such counselling? [Yes/No]

\section{Section F: Goal setting and discharge}

28. Do you collaborate with the following people in goal setting? (i) persons with aphasia, (ii) Friends/Family of persons with aphasia, (iii) Multidisciplinary team [Always/Frequently/Occasionally/Rarely/Never]

29. What are your three most common reasons for discharging persons with aphasia?

30. Do you review your persons with aphasia client after discharge? [Always/Frequently/Occasionally/Rarely/Never]

31. On average, when do you review your persons with aphasia clients after discharge? [ 2 weeks- 1 month $/ 2-3$ months $/ 6$ months/ 1 year/> 1 year]

32. What is done during the review session (tick all that apply)? [Review of language ability/Training of language ability/Communication partner training/Other (please state)]

33. After discharging persons with aphasia, did you think they still required language intervention? [Yes/No]

34. Were recommendations for service given to the persons with aphasia upon discharge? [Yes/No-Go to Q36]

35. What recommendations do you give usually for persons with aphasia upon discharge (tick all that apply)? [Home practice/Other private services/Educational materials/Workshops/Self-help groups/Support groups/Other (please state)]

36. Do you work directly in the community to improve communication access (e.g. educating bank or café staff about communicating with persons with aphasia)? [Always/Frequently/Occasionally/Rarely/Never]

37. Do you provide education to health practitioners regarding how to communicate with persons with aphasia? [Always/Frequently/Occasionally/Rarely/Never]

38. What would you like to see in future research regarding treatments for aphasia? 